\title{
Probing the Potential of Post-Anthropocentric 3D Printing
}

\author{
Laura Devendorf $^{1}$, Abigail De Kosnik ${ }^{2}$, Kate Mattingly ${ }^{2}$, Kimiko Ryokai ${ }^{1}$ \\ ${ }^{1}$ School of Information \\ UC Berkeley \\ Berkeley, CA, USA \\ \{ldevendorf, kimiko\}@berkeley.edu \\ ${ }^{2}$ Theater, Dance, and Performance Studies \\ UC Berkeley \\ Berkeley, CA, USA \\ \{adekosnik,katematt\}@berkeley.edu
}

\begin{abstract}
The growth of small scale manufacturing technologies associated with the "maker movement" has captured the attention of artists, innovators, educators, and policy makers. This paper critically examines how one core technology of the maker movement, a 3D printer, materializes assumptions about makers and their preferred ways of working with machines and materials. We describe how existing designs can be seen as anthropocentric, framing the human maker as visionary and commander of passive machines and materials. We then present an alternative system for 3D printing, called Redeform, which explores how a post-anthropocentric framing of makers as collaborators with machines and materials changes the design of 3D printers. We place our system within a lineage of performances that have explored relationships between humans and nonhumans since the 1950s. In doing so, we explore and speculate on the opportunities for operationalizing post-anthropocentric theories within the specific context of the maker movement.
\end{abstract}

\section{Author Keywords}

Maker movement; digital fabrication; new materialisms; indeterminacy; resistance; performance art.

\section{ACM Classification Keywords}

K.4.0. Computers in Society: general.

\section{INTRODUCTION}

The so-called "maker movement" has emerged from the combination of new developments in grassroots manufacturing technology with a body of potential users eager to make their own products, designs, trinkets, and tools. Dale Dougherty, founder of Make magazine, attributes the growth of the maker movement to factors including "the introduction of new technologies such as 3D printing" and "hyper-local efforts to convene those who

Permission to make digital or hard copies of part or all of this work for personal or classroom use is granted without fee provided that copies are not made or distributed for profit or commercial advantage and that copies bear this notice and the full citation on the first page. Copyrights for thirdparty components of this work must be honored. For all other uses, contact the Owner/Author.

Copyright is held by the owner/author(s).

DIS 2016, June 04-08, 2016, Brisbane, QLD, Australia

ACM 978-1-4503-4031-1/16/06.

http://dx.doi.org/10.1145/2901790.2901879 share common goals" [11]. The technologies of the movement, specifically 3D printers, have captured the attention of artists, educators and policy makers alike. The appeal of $3 \mathrm{D}$ printing for artists includes the ability to physically realize unimaginable structures and forms created through generative coding. For educators and policy makers, exciting new machines for making things are looked upon as a means for motivating young students to pursue careers in science, technology, engineering, and mathematics.

A "maker" is someone who shares a "do-it-yourself" work ethic. Dougherty writes, "makers are seeking an alternative to being regarded as consumers, rejecting the idea that you are defined by what you buy" [11]. By identifying makers by a shared set of values rather than specific technical skills or practices, the rhetoric of the maker movement promotes inclusivity and suggests that the doors to the movement are open to all who share its do-it-yourself spirit. Often, "joining" the movement entails participation within a maker community, whether it is a digital community like instructables.com or a physical space like a shared machine shop, hacklab, or maker space. In the case of physical communities, access to fabrication machines serves as a locus to unite people of varying interests and backgrounds.

While most hacklabs and makerspaces claim to be neutral spaces for makers of all backgrounds and interests, the politics of participation are more complicated as some forms of working are seen to be more legitimate or technically sophisticated than others. Toombs et al. describe barriers to access in hackerspaces as "sociological," relating to how someone fits in with the existing culture of the space [29]. Feminist hacker spaces like Double Union in San Francisco can be seen as a response to sociological barriers of access. These spaces have come into being to address the specific interests and styles of working shared by women interests like identity workshops or sketching that the founders of Double Union found to be marginal at traditional hackerspaces $[12,16]$.

Reflection on participation in the maker movement has tended to focus on the accessibility of particular technologies or the social dynamics of physical spaces. We shift focus to the technologies of making and examine how their workflows embody and reinforce a particular set of values in making. Specifically, we examine how the design of a $3 \mathrm{D}$ printer, a technology that is central to the rise of the 
maker movement, allows for particular values in design, like accurate replication, to be expressed while making other values, such as close interactions with materials, more difficult.

\section{BACKGROUND}

\section{The Politics of 3D Printers}

Scholars in science and technology studies and related fields have described the how technological artifacts express politics and materialize the ethics and viewpoints of the stakeholders who contribute to their design [21,31,32]. Critical technical practice is an approach developed by Agre to surface the politics embedded in designs and to describe their effects on human experiences [1]. As an exercise in critical technical practice, this paper unpacks existing assumptions about makers that are built into the design of $3 \mathrm{D}$ printers and then reimagines the design by drawing from an alternative set of assumptions. Specifically, we identify existing designs as anthropocentric and we draw from theories associated with the new materialisms [7] in order to suggest a post-anthropocentric design for a 3D printer. Our goal is not to offer a design to replace existing 3D printers or to suggest incremental improvements to existing designs, but to highlight the biases of 3D printers: the kinds of making that are more or less difficult and the relationships with materials that are and are not supported. For instance, existing 3D printer designs make it difficult to physically manipulate a structure as the machine is building. One could argue that such a design is biased towards users who value accuracy and efficiency rather than those who prefer a process that recognizes the value of errors in disclosing new approaches. By calling existing design norms into question, we offer a lens through which one can reflect on the relationship between fabrication systems design and participation in the maker movement. We shed light on how a designer's conceptualization of the value of making is enacted in design and how these value judgments ultimately reinforce or challenge norms around what it means to be a "maker."

\section{The Changing Landscape of Fabrication}

Researchers in and out of HCI have developed innovative fabrication technologies that allow makers to work with computationally specified instructions without compromising close engagements with materials [10,26$28,35]$. This paper analyzes one such technology, Redeform (previously named "Being the Machine" [10]), to provide a broader theoretical framework for thinking about how such projects shape relationships between makers, technologies, and materials.

This paper synthesizes and expands upon two previous projects. The first, titled "Being the Machine" presented technical descriptions and accounts of user experiences with what we refer to here as Redeform [10]. We highlighted how dealing with resistance and/or unpredictable materials was a core value in the practices of many makers and argued that technology can play other roles in making beyond preventing failure and producing accurate models. The second was an exploration of how themes expressed in performance art could be engaged in the design of fabrication systems [9], in order to highlight how situated actions, in addition to objects, can be the locus of meaning in fabrication activities.

This paper offers a deeper reflection on the connections between new materialist theory, post-WWII performances, and fabrication systems design that have emerged out of Devendorf's own artistic practices with Redeform over the past 20 months. In doing so, this piece takes a step back to reflect on how HCI thinks about values of makers and making and how those values inevitably shape the technologies we build. The goal of this work is to provide researchers with intellectual resources that help them critically examine the way digital fabrication systems structure relationships between humans, machines, and materials. Since enthusiasm about the maker movement is already impacting education and culture, attention to the politics and priorities embedded in this movement need to be analyzed and acknowledged in order to ensure that a diversity of making systems and making styles are being offered and honored.

In the paper that follows, we summarize two contrasting theoretical positions, hylomorphism and morphogenesis, to understand what making is and the specific ways in which forms come into being through making. We describe how existing 3D printer designs can be seen as hylomorphic and draw from a contrasting theory of morphogenesis to suggest a new kind of 3D printer. We discuss our design within a lineage of performance art in order to paint a speculative vision of the experiential and aesthetic implications of interacting with a fabrication system designed through the lens of morphogenesis. Drawing from our design, performance processes, and new materialist theory, we conclude by describing the kind of maker we aim to support, the particular values they may share, and how we might make room for these values in the maker movement.

\section{ANTHROPOCENTRISM AND 3D PRINTERS}

While making always results in a form, various theories describe how that form comes into being. Some theories can be classified as anthropocentric, framing the human maker as the primary factor determining form while others can be described as post- or non-anthropocentric, framing form as something that emerges from complex negotiations between humans, tools, materials, and environments. Hylomorphism is an anthropocentric theory in which form is believed to be determined independently of material forces [8,18]. In anthropologist Tim Ingold's terms, "Whenever we read that in the making of artefacts, practitioners impose forms internal to the mind upon a material world 'out there', hylomorphism is at work" [18]. A hallmark of hylomorphism, and anthropocentrism more broadly, is the belief in the passivity of matter. With passive 
matter, an idea can be faithfully translated into an object with little change arising within the process of translation.

To understand 3D printers as hylomorphic, one must first understand how a 3D printer works. A 3D printer is a kind of machine within a broad category of computer-numeric controlled (CNC) machines. CNC machines and their associated software translate digital models into a list of instructions that a machine can perform to create a physical version of that digital model. The language used to specify the movements (what are technically referred to as toolpaths) of these machines is called G-Code. G-Code offers a standard set of codes to tell machines things like where to move, how fast to move, and, depending on the machine, where to add or remove material. Creating the mechanics and algorithms for translating a digital model into a list of G-Code instructions intended for a particular machine is a key design challenge when developing CNC technologies. Currently, the heuristic that has driven development in CNC machines, including 3D printers, has been fidelity to the original digital model.

The focus on accurate replication of a pre-existing model makes the designers of CNC systems seek to eliminate all sources of uncertainty that may potentially alter the form from what has been specified digitally. In other words, it drives designers to identify methods to tame the material world so that it will passively take on any shape or form. Such a vision of passive materials is reflected in the rhetoric of engineer and maker movement evangelist Neil Gershenfeld, who imagines that, "personal fabrication will bring the programmability of the digital worlds we've invented to the physical world we inhabit" [13]. While this vision has certainly ushered in powerful new innovations, forms, and processes of creation, it is also one that frames the human maker as the locus of innovation and creativity and their building materials as passive receptors or containers for makers' ideas - an idea we believe limits the design space of 3D printing for two primary reasons.

First, commercial 3D printers tame the material world by limiting building materials to those that have been engineered to readily take on many shapes and forms. For example, MakerBot ${ }^{\circledR} 3 \mathrm{D}$ printers are designed to work specifically with acrylonitrile butadiene styrene (ABS) or polylactic acid (PLA) filaments. One of the primary advantages of $3 \mathrm{D}$ printing is the ability to realize a form that was created digitally, perhaps using generative computational means to create once unimaginable forms, and to use that form as a basis for the production of a material object. Yet, the flexibility to change structures and forms with code or computer aided design tools is far greater than the flexibility one has when the design "leaves" the digital world so to speak and enters the physical world. The physical world is full of materials with broad ranging shapes, properties, behaviors, and textures that can be engaged in making to lend particular qualities to the form produced. By embracing the unique and lively characteristics of everyday materials, from sticks and leaves to candy or carrots, we see an opportunity to produce a set of hybrid computational-material forms with textural, aesthetic, and symbolic characteristics vastly different than those created from filaments used in traditional 3D printers.

Second, attempting to pacify building materials for the sake of accurate replication limits the experiential range of $3 \mathrm{D}$ printers. Currently, the idealized experience of commercial 3D printing involves setting up the printing environment and hitting "go," at which point the machine takes over and the human is free to pursue other projects. While such an approach is ideal in terms of efficiency and multitasking, it removes the human from the process of making, preventing them from developing sensitivity to the materials though touch and feeling them as they mold or resist particular forms. By developing a rich intuition about the way in which building materials adapt to $3 \mathrm{D}$ printing processes, one may begin to imagine novel ways of reworking both processes and materials in order to develop ideas for future forms. Furthermore, continued care, experimentation, and growth with a set of materials can form the basis of a sustained relationship with materials that many find pleasurable and even therapeutic. By augmenting 3D processes to accommodate rich sensory experiences, we see opportunities to extend the ways in which one can bring computation in tandem with physical materials.

As we describe the limitations of hylomorphic design ideals, it is important to consider that while technology shapes interaction, it does not script or specifically determine the way in which a maker might think, feel, or do certain things [22,33]. Accordingly, a CNC machine designed from a hylomorphic perspective does not imply that a maker's actions with the machine could also be described as hylomorphic. All interactions with machines are constrained and users are always negotiating their desires with a machine's functionality. What most makers describe as a "successful" 3D print, in the sense that it produces what they expect, emerges only after multiple attempts, tweaks, and adjustments have been made to control unpredictability on the users' end. For instance, the ABS plastic filaments used by many $3 \mathrm{D}$ printers tend to curl in on themselves at sharp corners, requiring the user to add extra support structures or "helper discs" to her model to keep the corners firmly affixed to the building surface. Additionally, the heat sensitive materials used for 3D printing act less predictably when the temperature of the environment is not controlled, say, when the printer is placed near a sunny window.

To summarize, while a designer's imagined workflow of $3 \mathrm{D}$ printing may be hylomorphic, the reality of 3D printing is anything but: 3D printing, like all craft, forces the maker to contend with stubborn recalcitrance and unpredictability of the material world. A non-hylomorphic design approach frames material recalcitrance and unpredictability as beneficial aspects to explore within interactions with the 
technology, rather than aspects to be eliminated by technology. It shifts human labor from attempting to control the printing environment to sensing the materials and finding new and exciting ways to open the production process to material forces. For instance, melting, which might be caused from a printer being too close to a sunny window, can shift from being regarded as a problem or nuisance to new and interesting force that offers a potentially beautiful dripping texture to be crafted.

There are many ways that makers can (and do) embrace the recalcitrance and unpredictable aspects of existing 3D printers. Thus, a non-hylomorphic design approach may offer different benefits for different audiences. For people who may not see resistant or recalcitrant materials as creative resources, non-hylomorphic designs could scaffold workflows that may change their perception. For people who already value working with stubborn materials, nonhylomorphic designs create spaces for unique encounters with digital and physical materials that might not have emerged from explorations with existing 3D printers.

\section{TOWARDS MORPHOGENETIC MACHINES}

As we stated above, a non-hylomorphic 3D printer frames building materials as entities to be harnessed and adapted in order to discover new forms rather than facilitators for realizing our pre-existing ideas. A desire to work with stubborn materials in fabrication is represented by a growing number of hacked or self-built 3D printing systems that use uncommon materials $[23,30]$ or playfully derivate from input models [20]. We see these actions as evidence for a desire to work beyond a model of human mastery over machines, since these actions seek a more open ended, even collaborative relationship with a rich set of materials and machines. Such an approach to design could be described as morphogenetic.

Morphogenesis offers a contrasting theory to hylomorphism: one in which form is not determined a priori, but emerges from a nexus of activity between human and nonhuman actors [8,18]. Drawing from Deleuze's use of the term, DeLanda writes, "We may now be in a position to think about the origin of form and structure, not as something imposed from the outside on an inert matter, not as a hierarchical command from above as in an assembly line, but as something that may come from within the materials, a form that we tease out of those materials as we allow them to have their say in the structures we create" [8]. Materials, in this view, take an active role in determining the form that emerges. Tim Ingold relates morphogenesis specifically to the way in which humans make things and uses the term "correspondence" to describe a relationship between humans and their nonhuman tool and material counterparts [18]. In the process of making, humans enter into correspondence with materials as well as the broader world within which they are working. Materials "speak" in this correspondence through their physical properties, pushing towards and pulling against a maker's actions.
Ingold argues that form can never fully be determined, only anticipated by a maker working in correspondence with his or her materials.

Thus far we have argued that hylomorphism in design can be limiting and suggested a morphogenetic perspective in design as a way to embrace the "activity," unpredictability, and stubbornness of materials in making. As the examples above show, some creative practitioners have already embraced such an approach in the design of 3D printing systems for their personal use, though not calling their design approach morphogenesis as $\operatorname{such}^{1}$. In the following section, we will describe a system we built in order ask how the specific theoretical framing of morphogenesis and concept of correspondence might push the design of 3D printers in an even more radical direction, uncovering new formal and experiential spaces that emerge through indeterminate engagements with machines and materials in specific spaces. By placing this work in relationship to the maker movement, we explore values in making that may be uniquely addressed by such an approach.

\section{REDEFORM}

Designed by Devendorf and Ryokai, Redeform (formerly "Being the Machine" [10]) is a system that asks humans to receive, interpret, and execute the G-Code instructions typically provided to a $3 \mathrm{D}$ printer in order to construct new objects, reversing the scenario of 3D printing, in which humans provide instructions to a printer that the machine must carry out. In essence, Redeform makes the human user into a 3D printer. Technically, Redeform consists of a laser pointer that illuminates a single dot on a surface. The dot moves along the tool-paths generated from a digital model that the human has inputted, showing the human how a 3D printer would lay down material and inviting them to follow by hand. For the human, this feels like a game of connectthe-dots. Upon completion of the task "described" by the laser pointer, the human has sculpted a three-dimensional object more or less like the digital model they specified. The materials used by humans to craft objects with the Redeform system have ranged from flowers to Cheez Whiz to pipe cleaners to balloons.

To use Redeform, the user creates or selects a 3D model that she would like to use as a starting point for interaction (figure 1a). The 3D model is processed through an open source G-Code generator called Slic3r [21] to convert it into a list of machine instructions. G-Code instructions for

\footnotetext{
1 The concept of morphogenesis and its relationship to $\mathrm{CNC}$ manufacturing technologies has been a growing area of exploration in architecture. Much of this work applies concepts of morphogenesis to the architectural design processes and makes use of existing $\mathrm{CNC}$ technologies to realize those designs. In this article, we advocate a similar approach in relation to interaction design, asking how morphogenesis can be mobilized to foster new kind of technology and experiences with those technologies.
} 

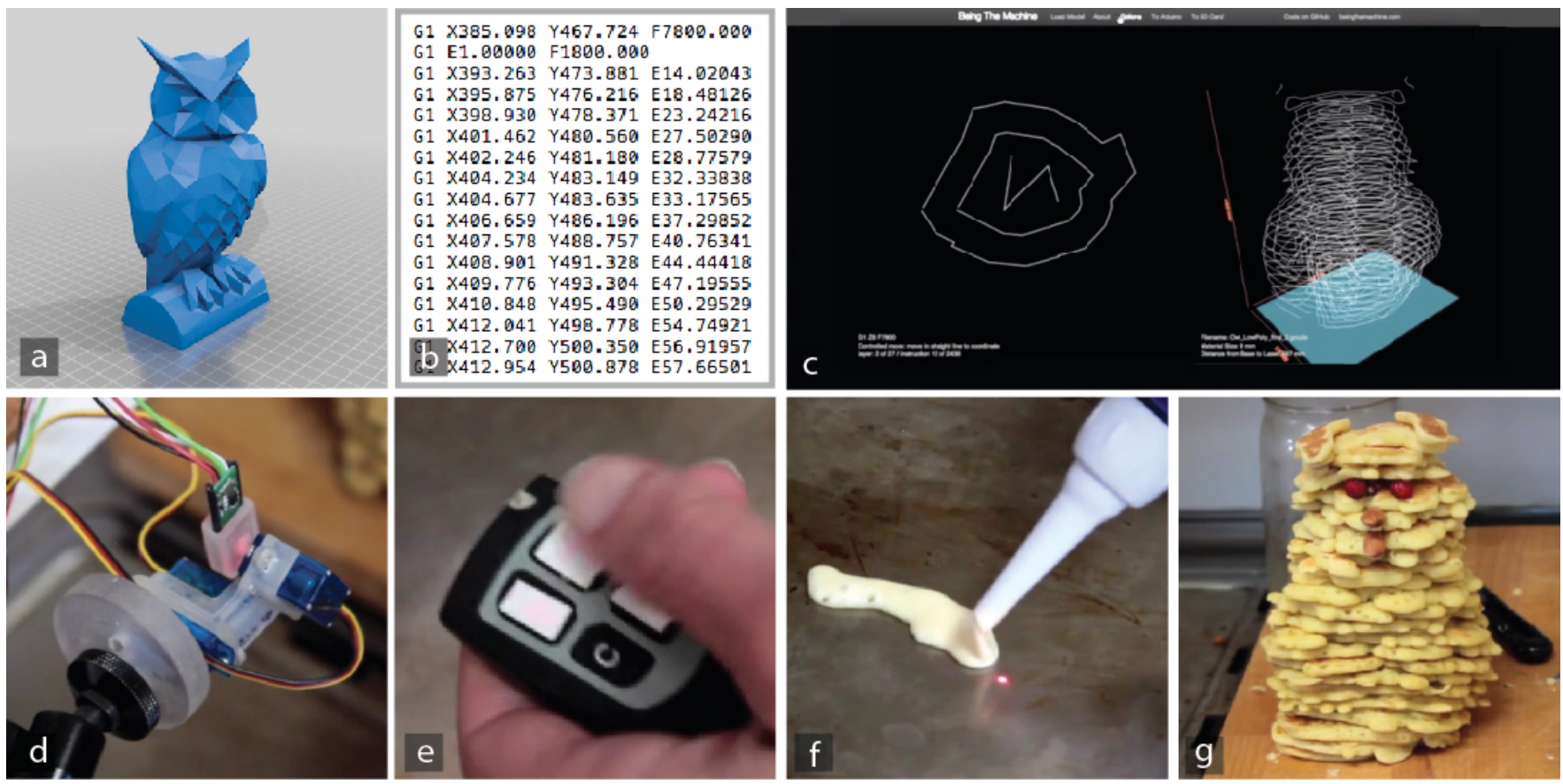

Figure 1: Working with Redeform. a. A 3D model of an owl is selected. b. The model is converted into G-Code. c. Visualization software allows the user to view the G-Code paths generated. d. Once the user finds the paths suitable, she can upload the G-Code to the Redeform machine, a laser pointer attached to 2 motors in a pan-tilt configuration. e. She presses buttons on a key-fob to tell

the machine to play or stop playing through machine instructions. f. She sees instructions as a single laser point and follows the

point with pancake batter on a hot skillet. g. After she follows all the paths described by the laser pointer, an object emerges.

many 3D printers are created by virtually slicing a 3D model into layers and converting each layer into a series of paths that can be drawn with a mechanical extruder (figure 1b). A 3D printer draws the layers one on top of the other to recreate the object. When translating a model to G-Code with Slic3r, the user is able to specify parameters such as material height and width, which affect the way the G-Code paths are specified. We created software that allows the user to visualize the G-Code paths generated from Slic3r so that she can get a sense of the movements that the model will be asking her to perform (figure 1c). After creating a G-Code file to her liking, she uploads it to our machine (figure 1d). She interacts with the machine using a key-fob with buttons that allow her to play forward, play backward, and stop the machine (figure 1e). If she pushes the play forward button, the laser point begins drawing from the current G-Code instruction to the next subsequent G-Code instructions. The user experiences this by seeing the laser point move along a path. Playing backward allows the user to do the same action in reverse. Pressing the stop button allows the user to stop the laser at its current position. By following the laser point with her materials (figure 1f), the user produces a series of paths and layers that begin to look more or less like her input model (figure 1g).

After conducting several user studies with Redeform [10] we found that one key design feature encouraged users to embrace qualities like indeterminacy and emergence characteristic of a morphogenetic perspective on making: following a single laser point. Redeform could have been designed using an alternate set of tools and techniques. For instance, a projector could have projected an entire outlined path, rather than a single point, which would have been easier to trace accurately. We made a strategic design decision not to use a projector for three primary reasons.

First, we wanted to encourage interpretation and emergence, not replication. A closed path tends to connote a need to be precisely copied or filled in, but a single moving point has no history and no future, allowing the user to follow it without the ability to compare whether or not they followed the point accurately. Second, we wanted interaction with our machine to provide the user with a bodily sense of the mechanics of 3D printers. Guiding a user along a path using a single point encourages the movements of the body to mimic those of the machine. Additionally, it prompts the user to work with her building materials in an uncommon way. By asking the user to do something strange with her materials, we aimed to heighten her sensitivity to the ways in which materials conformed to or resisted the machine paths. Third, we felt it was important for the machine to have a unique persona and performance of its own. The machine we created consists of a laser pointer attached to two motors in a pan-tilt configuration. When the user presses "play" on the machine, the machine begins to animate the motors and laser pointer. The gears of the motors produce a low humming sound unique to mechanical parts. The sounds and motions of the machine may not play a primary role in interaction, but they become peripherally present as part of the environment of making. 

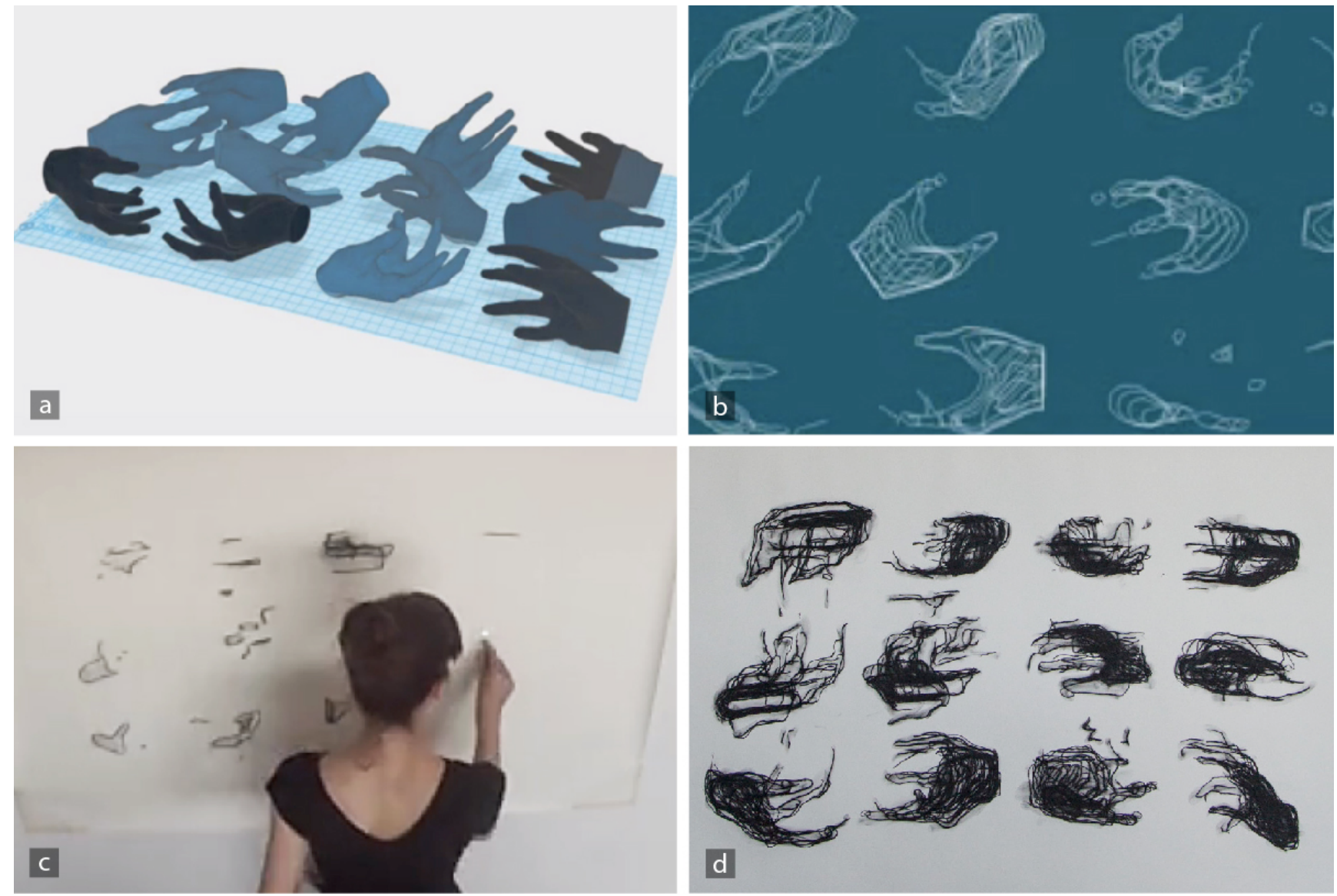

Figure 2: Following Redeform with Charcoal. a. 3D model of several hands in different orientations is used as input to Redeform b. A visualization shows the tool-paths generated from the 3D model. $c$ The laser pointer moves along the tool-paths and Devendorf follows it with charcoal. $d$. The drawing that emerges is abstract derivation of the original 3D model.

By framing Redeform as a machine to be interpreted and experienced, we created a platform to sustain close interactions and growth with a large set of materials. The design itself contains affordances to suggest particular ways of working that de-emphasize precision in order to make space for new ideas, forms, and experiences to emerge. Additionally, the way in which both the materials and the machine become present to the user, through the machine's movements or the feeling of the materials on her hands, allows her to become entangled in the agencies of nonhuman actors. Redeform created a very different experience of 3D printing and set the stage for a mode of making characterized by improvisation and discovery.

\section{Redeform in Practice}

The way a maker's sensitivities change and materials become activated within interactions with Redeform is best illustrated through example. For the past 20 months Devendorf has been using Redeform as part of her personal art practice. In January 2015, she began to experiment with shapes and forms that emerged as she followed the laser with charcoal on paper. Experimentation entailed generating digital models, translating them into G-Code files, uploading those G-Code files to the laser guide, and then following the laser guide by hand with charcoal. At every step in the processes a different set of digital and physical materials became activated, causing the model to deviate from its previous form. For instance, the digital input model is manipulated by the parameters specified to the G-Code generator, the motors which drive the laser guide have limited resolution and rasterize the paths Devendorf follows, and, Devendorf's own hand imperfections modify the paths even further.

Experimenting with the system led to growth with both digital and physical materials. After several experiments with 3D models of hands, Devendorf began to realize how arranging the hands in different orientations in $3 \mathrm{D}$ space produced radically different looking tool paths. While early experiments focused on drawing a single model, she felt the drawings to be more visually and conceptually interesting if the same model was repeated multiple times in the composition because it visually emphasized tensions between replication and derivation. Additionally, Devendorf began to develop new ways to work with her "building" material, charcoal. Charcoal has a unique capacity to smear on paper and in early experiments Devendorf found herself trying to limit smearing by carefully keeping her hand lifted off the page. A fellow 
artist offered the critique, "the charcoal isn't doing anything for you in these drawings," which she took to mean that she was not embracing the properties of charcoal that make it uniquely different from something like a marker or pencil. In subsequent experiments, she embraced the smearing nature of charcoal by actively dragging the side of her hand as she drew. The results were striking. Since drawing flattens the tool-paths for a 3D object into a 2D plane, the degree of smearing became a way to represent the layers of the model, with darker regions representing areas shared by many overlapping layers, and lighter regions representing areas with less layering.

Devendorf experienced growth with materials across the entire process. For instance, realizations about the behaviors of charcoal affected the way in which digital models were constructed and vice versa. The ongoing negotiation and development of all parts of the system could be described as correspondence. In use, Devendorf is not only corresponding with paper, charcoal, and the machine, but also the digital models, customization software, and hardware that comprise the machine.

Interactions with Redeform also called attention to materials that were not originally engaged in the process of making. For instance, when exhibiting the project, Devendorf preferred to perform the laser-guided drawings live in front of a gallery audience. The act of performing the machine made Devendorf consider how her physical movements in space became choreographed by the toolpaths and led her to change models and G-Code in a way that would produce movements that may be interesting for viewers. At one performance, the laser guide was mounted to an overhang, which extended from the second floor of the building. The vibrations of both the amplified music as well as the footsteps of people walking on the second floor shook the laser point Devendorf was following and became represented in the drawing. Additionally, many attendees began to engage Devendorf by asking her about the system and the way it worked. Often, the attendees would stand in front of the laser point, unaware that their body was occluding the mark that Devendorf was following. Rather than pausing the system and waiting for the attendee to move or moving the laser guide to a more stable location, Devendorf began to see the people and vibrations of the space as additional active forces in the drawing process: their presence being represented by shakes and gaps in the traced paths. The result from each performance was a completely unique drawing, not simply because Devendorf's hand movements are unique, but because the dynamics of the space, its vibrations and people, all became captured in the marks on the paper.

While Redeform guided Devendorf in doing things like a machine, it would not be correct to say that Redeform is ultimately controlling interaction. Such a strict binary between human and mechanical agency would deny the many layers and filters of agency that become enmeshed in interaction. For instance, Devendorf chose the model she would eventually draw, she controlled the speed of the laser, and to some degree, the way the G-Code paths were generated. Furthermore, there are contributors to the interaction that extend beyond the Devendorf and Redeform, for instance, the vibrations of the speakers that shook the paths in her performance. To say that the machine is specifically "controlling" interaction would make as much sense as saying a coloring book is controlling the way in which someone chooses to color. We prefer to think of Redeform as a way to shape, but not determine, interaction towards a particular set of effects. With Redeform, Devendorf chose specific constraints in order to bring herself and audiences viewing her performances to a place to understand and reflect on human-machine relationships, to draw these experiences in contrast to other experiences with $3 \mathrm{D}$ printers, to reflect on the way that all technologies can be both freeing and restricting, and to create a decidedly strange experience that can open up new perspectives on familiar actions.

\section{Redeform and an Indeterminate Tradition}

Through Devendorf's experiences with Redeform, we began to tease out how the system set the stage for both human and nonhuman actors to perform. Devendorf was performing in a literal sense when she created drawings for an audience but her human performance was choreographed within and around the performances of the nonhuman actors: the charcoal, machine, and vibrations in the space. By framing these nonhumans as actors rather than tools or media to be acted upon, the agency of nonhumans became foregrounded in interaction allowing for new kinds of processes, forms, and understandings to emerge. We began to see connections between the kind of making we created by operationalizing morphogenesis in design and the kind of making taking place within chance-based and participatory art practices, which gained prominence between 1950 and 1980. In the following section, we reflect on this tradition of art making in order to unpack the meaning and values embodied in Redeform, as well as the aesthetic and personal impacts morphogenetic maker technologies may have more broadly.

In the 1950s, experimental composer John Cage founded an approach to art making that featured co-operations between human players and nonhuman objects that combined to generate sound in unpredictable and surprising ways. In Cage's "Water Walk" [5], Cage assembled a large number of ordinary household appliances, including five unplugged radios, an iron pipe, a bathtub, a soda siphon, an ice bucket, a vase with flowers, a tape machine, a garden sprinkler, a pressure cooker. Cage then walked from object to object, using each in its commonplace way, and the sounds created by his "playing" each thing produced a vibrant cacophony that was wholly different than anything a musician might have played on a musical instrument. In Cage's work, the musician is not someone who occupies the role of "master" 
or who controls and dominates "servant" instruments; rather, the performer aims to activate latent potentials in everyday objects at hand, and is ready and open to the soundscapes that result from her aleatory interactions with these nonhuman collaborators. The humans and the nonhumans, in this piece and in many of Cage's works, form an assemblage in which a human does not have hierarchical superiority, but all elements form a reciprocal relation and act equally on one another. Cage's methods proved to be highly influential on generations of artists' practices.

In 1965, choreographer Yvonne Rainer developed a piece called "Parts of Some Sextets," which consisted of Rainer's 10 performers moving 12 mattresses around a stage in multiple configurations and interacting with them in playful and seemingly bizarre ways. For example, Rainer's instructions include these scenes:

...18. Human flies on mattress pile; 19. Formation \#1 (fling); 20. Formation \#2 (with 'bug squash'); 21. Move pile to other side.... [24].

Rainer's conception for "Parts of Some Sextets" calls the performers' and the viewers' attention to the possibility that objects can create choreography as opposed to a human choreographer planning each step of a piece. Human performers in this work do not wholly dominate the mattresses, nor do the mattresses completely dominate the humans; rather, the humans and nonhumans exchange power and alternate in the roles of "actors" and "acted upon."

A contemporary of Rainer, choreographer Trisha Brown, designed "Walking on the Wall" in 1971, which similarly presented human performers as partners (rather than controllers) of nonhuman materials [4]. This project featured seven dancers attached by harnesses to a system that allowed them to walk on the walls, their heads pointing towards the center of the room, so that the performers' bodies were parallel to the floor as they walked. Brown recognized that there would be a high degree of difficulty for the dancers attempting to maintain equilibrium between gravity and the force of the harness/pulley system as they walked on the wall, and in all of the performances of "Walking," the dancers do not mask their effort, or make the piece seem polished or "finished." Instead, the performers in 1971 wore their ordinary workout clothes (not costumes), and did not move to any music-their dialogue with one another, and the sound of the harness/pulley system, were the only sound cues. The "working out" of the performance constituted the performance. There was no completed piece that was refined through many rehearsals and only shown to the public when it had achieved a degree of "readiness." In addition, Brown's work exposes the apparatus of making: the pulleys, ropes, and harnesses were fully visible throughout the performance, and even the surface of the wall, which substituted for the dance floor, was made prominent and served as a contributing and determining element in "Walking." Thus, "Walking" foregrounded the collaboration of human performers and nonhuman materials, and honored the generative accidents that arose from such interactions of people and objects.

At the same time that Cage, Rainer, Brown created the pieces described above, the artists' network called Fluxus formulated a theory of what they called "intermedia art" [17]. Intermedia art is defined by the co-presence and interdependence of humans and objects-especially machines, such as film projectors - in performance spaces. The Fluxus group aimed to create intermedia art that heavily emphasized indeterminacy in the collaboration of disparate elements (for example, one of their books was titled An Anthology of Chance Operations [34]). We regard Redeform as an intermedia art construct, which brings humans into relation with machines (a computer and motorized laser guide) and other nonhuman matter (the substances that the humans use to build their pieces, such as pancake batter or charcoal), in a way that does not frame the human as a "user" nor the nonhumans as "media," but establishes a more equal power dynamic across all of the participants or members of the relation, and leaves the outcome of the relation open to chance and accident.

In Redeform, as in the aleatory works of Cage, Rainer, Brown, and the Fluxus group, the human performer does not make use of a nonhuman medium, but serves as the medium through which performers and objects create something collaboratively, causing a new form to come into being. The human in Redeform is not wholly the servant of the machine-the human decides what to program the machine to tell them to do, after all-but after the human has programmed the machine with some instructions, the human then becomes a servant of the machine, using eyes and hands to follow the machine's instructions as best as they are able. The human's inability to "perfectly" execute the machine's directions, as well as any resistance to manipulation shown by the substance that the human is trying to shape into an object, reveal that the true "product" of Redeform is, in fact, a process - the process of labor coperformed by human and nonhuman makers.

\section{DISCUSSION}

Drawing from our experiences designing, working with, and studying Redeform, as well as aesthetic potentials expressed by the performances discussed above, we conclude with a speculative vision of the impact morphogenetic design, a particular post-anthropocentric design perspective, could have on maker technologies and the maker movement more broadly. While we advocate for open-ended and emergent approaches to making, we are not suggesting that precision and accuracy are bad or that all CNC systems should embrace unpredictability. Rather, we argue for an expanded view of values in making that regards control-driven (hylomorphic) and indeterminate (morphogenetic) systems as valuable in different contexts. 


\section{Decentering the User in Design}

Commercial 3D printers, and most consumer facing technologies for that matter, aim to place the user in the "center" of design, and thus, in a role to command machines and materials. The user is positioned above and apart from the technology and the technology does its best to offer intuitive controls, seamlessly supplying the user with the information or resources she requires. Redeform and the performance works outlined above place humans and nonhuman machines and materials on more equal footing. In Redeform, the technology is strategically nonintuitive and gets in the way of its user, forcing her to negotiate her goals with the machine. In morphogenetic design agendas, we see the user transitioning from a commander of materials to an orchestrator of materials, placing materials in various configurations and arrangements to produce a range of non-specific, but nonrandom, outcomes. As the user shifts from commander to orchestrator, her position is no longer "central" as other human and nonhuman forces can challenge and resist her actions. Thus, she loses the ability to predict the outcome of making but gains an opportunity to discover surprising or unexpected forms, insights, and expressions that emerge only by limiting her voice in the creative process.

DiSalvo and Lukens also describe how post-anthropocentric theories (namely, Actor-Network theory and Object Oriented Ontology) decenter the human user in design [6]. They developed technologies that allow humans to see through the perspective of robots, and highlighted how this can lead to new insights, understandings, and sensitivities to those perspectives. Redeform and the performances described above reveal how decentering the maker or artist can create provocative engagements that foreground the dependencies between, and mutual shaping of, humans and technology.

Consider Karen Barad's term “intra-action” to describe,

...the mutual constitution of entangled agencies. That is, in contrast to the usual 'interaction,' which assumes there are separate individual agencies that precede their interaction, the notion of intra-action recognizes that distinct agencies do not precede but rather emerge through, their intraaction [2].

With this definition in mind, we see hylomorphic design perspectives as those that hold human and machines as categorically different, with assumed agencies that precede interaction. Alternatively, we see morphogenetic designs supporting intra-actions among machines, materials, audiences, and environments. Within intra-actions with materials, machines, software, and people, the maker is able to affect and be affected by the agencies of all the forces and factors involved. By blurring roles of human and machine, or media and tool, these technologies can make room for new roles, agencies, and outcomes to emerge. In the case of Redeform, boundaries between human and machine become blurred by allowing a human to act like a machine. In intra-action, human makers are provided with an opportunity to reflect on and even tinker with their positioning and power dynamics among the other forces involved in the making process. Thus, the process of making transforms from a way to produce things to a way to inquire about relationships among things, spaces, people, and materials.

This new configuration of making-less about a human subject and more about the potentials that inhere in collaborations between humans, materials, and machinesaligns with posthumanism as defined by N. Katherine Hayles [15]. Hayles' theories of posthumanism attempt to de-center the human "self" as the master of technological systems. She proposes that humans must be seen "as part of a distributed system" incorporating both human bodies and machine bodies, human intelligences and machine intelligences, and that "the full expression of human capability can be seen precisely to depend on the splice" between humans and technologies. Post-anthropocentric making technologies operate within this "splice" and highlight contingencies between human and nonhuman agencies, opening them to reflection and experimentation.

\section{Making with Vibrant Materials}

Political theorist Jane Bennett argues that recognition of the agency of nonhuman things can foster more sustainable behaviors in the world. Bennett's "vital materialism" calls attention to the livelihood of nonhuman things and their ability to affect us in "not-quite-human" ways. She writes,

The capacity to detect the presence of impersonal affect requires that one is caught up in it. One needs, at least for a while, to suspend suspicion and adopt a more open-ended comportment. If we think we already know what is out there, we will surely miss much of it [3].

Designing machines for making, like 3D printers, from a morphogenetic perspective is a particularly useful way for allowing users to become "caught up," or as Ingold might say, "join forces," with the affects of other human and nonhuman forces. In these encounters, strange constraints requiring one to build like a machine or cues that connect the actions of people and mattresses become an asset, allowing users to break away from familiar associations with things in order to see, create, and experience them anew. Humans can begin to identify with their nonhuman counterparts and may begin to seek alternative ways of working with materials as opposed to acting on them. By creating modes of making that make the "voice" of nonhumans louder, as in intra-action, these machines can be seen to have sensitizing capacities. They foster close, careful, and attentive relationships among humans, machines, and materials. Morphogenetic making machines, then, are well suited to "slow" [14], reflective engagements with machines and materials as opposed to rapid, productdriven prototyping. 


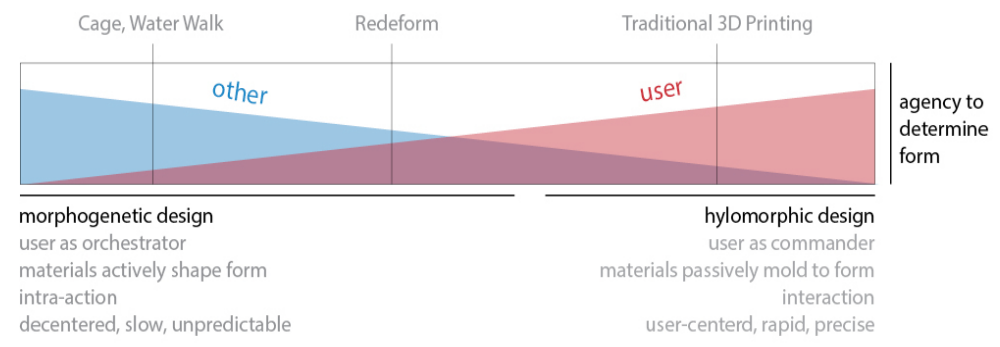

Figure 3: A high-level sketch of the differences between the morphogenetic and hylomorphic design perspectives.

\begin{abstract}
A Post-Anthropocentric Maker and Movement Post-anthropocentric maker technologies formulate an image of a post-anthropocentric maker who becomes innovative and technologically fluent by working closely with machines and materials, listening and sensing their actions, and responding accordingly. For this maker, empowerment is not about the ability to realize one's existing ideas, instead, it stems from the ability to listen and respond to materials as they take on forms. This maker is a performer among a cast of human and nonhuman counterparts, organizing her actions in relation to the others to form critical inquiries into the world. While the machines, materials, and outcomes may look similar to other acts of making, the way this maker goes about doing making may be better understood as improvisation, interplay, and entangled performance rather than as predetermination, domination, and control.
\end{abstract}

What separates anthropocentric from post-anthropocentric making is a matter of perspective. Two makers may use the same machines and materials but the way in which each maker relates to her materials can be quite different. Additionally, a single maker may desire different modes of making at different points within their practice, say, a postanthropocentric approach to generate new ideas and an anthropocentric approach to create multiple objects from those ideas. It is common to see designers, artists, and craftspeople using post-anthropocentric approaches to spur new insights about familiar materials and inspirational ideas. For instance, Jackson and Kang descriptions of artists working with or obsolete technologies can be seen as a mode of making that attends to the agency of "junk" [19]. Thus, it's reasonable to say that a post-anthropocentric maker movement is already taking place. Yet, maker spaces and the media associated with the maker movement make post-anthropocentric approaches difficult to detect or color them as different than "Making." This may be due to the fact that much of this work takes place as a social and technical inquiry rather than (or in addition to) a desire to "do-it-oneself." While it might be tempting to let these practices mutually exist in different arenas, (say, postanthropocentric approaches in art studios and anthropocentric approaches in maker spaces) carving a place for open-ended and indeterminate practices within the broader maker movement could be beneficial, particularly for fostering a diversity of opinions and working styles within educational contexts and maker spaces.
We see two ways (but there are likely to be more) that indeterminate practices can gain visibility and respect within mainstream maker culture. First, designers could create and maker spaces could include technologies like Redeform that allow for computational workflows to be applied in improvisatory ways. The presence of this technology in maker spaces could validate such practices and may appeal to broader audiences. Second, documentation associated with the maker movement could highlight process over product. Currently, finished products are the focus of knowledge sharing in the maker movement and are often presented as a kind of recipe for others to reproduce. In this model, the processes of working that gave rise to the idea for that product or its successful implementation are obscured. Knowledge gleaned from post-anthropocentric making might be communicated better as stories of practice, which highlight shifts in perspective, moments of inspiration, and an evolving thought processes.

\section{CONCLUSION}

This article weaves a narrative connecting new materialist theory, interaction design, and performance histories in an attempt to articulate ways of thinking about engagement that could broaden the landscape of digital fabrication research and foreground the potential of postanthropocentric modes of design. We describe how postanthropocentric theories, which argue for the view of nonhuman matter as active or even alive, can be operationalized in design by providing a description of Redeform and contextualizing it within a broader landscape of experimental performances. In doing so, we shed light on the important role indeterminacy and resistance play in interactions that place humans and nonhumans on equal footing. We speculate on the potential impact such a design approach might have within the specific context of the maker movement - namely, that such an approach could provoke reflection on human relationships with technology and could foster more thoughtful, careful, or reflective modes of engagement with core Maker technologies like $3 \mathrm{D}$ printers.

\section{ACKNOWLEDGEMENTS}

Thank you to the Autodesk Aritst-In-Residence program for their support of Devendorf as she developed Redeform. Thank you also to Rosemary Joyce, August Black, Daniela Rosner, James Pierce, and all of their reviewers for their feedback. 


\section{REFERENCES}

1. Philip E. Agre. 1997. Computation and Human Experience. Cambridge University Press, Cambridge ; New York.

2. Karen Barad. 2007. Meeting the Universe Halfway: Quantum Physics and the Entanglement of Matter and Meaning. Duke University Press Books.

3. Jane Bennett. 2010. Vibrant Matter: A Political Ecology of Things. Duke University Press Books, Durham.

4. Trisha Brown. 1971. Walking on the Wall. Retrieved from http://www.trishabrowncompany.org/?page=view\&nr= 481

5. John Cage. 1960. Water Walk. Retrieved from https://www.youtube.com/watch?v=-koTMW95NZs

6. Carl DiSalvo and Jonathan Lukens. 2011. NonAnthropocentrism and the Non-Human in Design: Possibilities for Designing New Forms of Engagement With and Through Technology. In From Social Butterfly to Engaged Citizen Urban Informatics, Social Media, Ubiquitous Computing, and Mobile Technology to Support Citizen Engagement. MIT Press.

7. Diana Coole and Samantha Frost. 2010. New Materialisms: Ontology, Agency, and Politics. Duke University Press.

8. Manuel DeLanda. 2004. Material complexity. Digital tectonics: 14-21.

9. Laura Devendorf and Daniela K. Rosner. 2015. Reimagining Digital Fabrication As Performance Art. Proceedings of the 33rd Annual ACM Conference Extended Abstracts on Human Factors in Computing Systems, ACM, 555-566. http://doi.org/10.1145/2702613.2732507

10. Laura Devendorf and Kimiko Ryokai. 2015. Being the Machine: Reconfiguring Agency and Control in Hybrid Fabrication. Proceedings of the SIGCHI Conference on Human Factors in Computing Systems, ACM.

11. Dale Dougherty. 2013. The maker mindset. Design, make, play: Growing the next generation of STEM innovators: 7-11.

12. Sarah Fox, Rachel Rose Ulgado, and Daniela Rosner. 2015. Hacking Culture, Not Devices: Access and Recognition in Feminist Hackerspaces. Proceedings of the 18th ACM Conference on Computer Supported Cooperative Work \& Social Computing, ACM, 56-68.

13. Neil Gershenfeld. 2007. Fab: The Coming Revolution on Your Desktop--from Personal Computers to Personal Fabrication. Basic Books, New York.

14. Lars Hallnäs and Johan Redström. 2001. Slow Technology - Designing for Reflection. Personal Ubiquitous Comput. 5, 3: 201-212.

15. N. Katherine Hayles. 1999. How We Became Posthuman: Virtual Bodies in Cybernetics, Literature, and Informatics. University Of Chicago Press, Chicago, Ill.
16. Liz Henry. The Rise of Feminist Hackerspaces and How to Make Your Own. Model View Culture. Retrieved October 29, 2014 from https://modelviewculture.com/pieces/the-rise-offeminist-hackerspaces-and-how-to-make-your-own

17. Dick Higgins. 1966. Statement on Intermedia. Décoll/age (décollage) 6.

18. Tim Ingold. 2013. Making: Anthropology, Archaeology, Art and Architecture. Routledge, London; New York.

19. Steven J. Jackson and Laewoo Kang. 2014. Breakdown, Obsolescence and Reuse: HCI and the Art of Repair. Proceedings of the SIGCHI Conference on Human Factors in Computing Systems, ACM, 449458. http://doi.org/10.1145/2556288.2557332

20. Jeff Maeshiro, Jia Wu, and Mary Sek. 2014. Geoweaver. Retrieved from http://maeshirodesign.com/2014/07/05/geoweaver/

21. Bruno Latour. 1994. Where are the Missing Masses? The Sociology of a Few Mundane Artifacts. In Shaping Technology / Building Society: Studies in Sociotechnical Change. The MIT Press, Cambridge, Mass.

22. Bruno Latour. 2007. Reassembling the Social: An Introduction to Actor-Network-Theory. Oxford University Press, Oxford; New York.

23. Ron Rael and Virgiina San Fratello. Emerging Objects. Retrieved September 9, 2014 from http://www.emergingobjects.com/

24. Yvonne Rainer. 1965. Some Retrospective Notes on a Dance for 10 People and 12 Mattresses Called "Parts of Some Sextets," Performed at the Wadsworth Atheneum, Hartford, Connecticut, and Judson Memorial Church, New York, in March, 1965. The Tulane Drama Review 10, 2: 168-178. http://doi.org/10.2307/1125242

25. Alessandro Ranellucci. Slic3r. Retrieved from http://slic3r.org/

26. Alec Rivers, Andrew Adams, and Frédo Durand. 2012. Sculpting by Numbers. ACM Trans. Graph. 31, 6: 157:1-157:7. http://doi.org/10.1145/2366145.2366176

27. Alec Rivers, Ilan E. Moyer, and Frédo Durand. 2012. Position-correcting Tools for 2D Digital Fabrication. ACM Trans. Graph. 31, 4: 88:1-88:7. http://doi.org/10.1145/2185520.2185584

28. Studio Homunculus. Haptic Intelligentsia. Retrieved from http://studio-homunculus.com/portfolio/hapticintelligentsia-human-prototyping-machine/

29. Austin L. Toombs, Shaowen Bardzell, and Jeffrey Bardzell. 2015. The Proper Care and Feeding of Hackerspaces: Care Ethics and Cultures of Making. Proceedings of the 33rd Annual ACM Conference on Human Factors in Computing Systems, ACM, 629638. http://doi.org/10.1145/2702123.2702522

30. Unfold. 2012. Stratigraphic Porcelain. Retrieved from http://unfold.be/pages/stratigraphic-porcelain 
31. Peter-Paul Verbeek. 2011. Moralizing Technology: Understanding and Designing the Morality of Things. University Of Chicago Press, Chicago ; London.

32. Langdon Winner. 1980. Do Artifacts Have Politics? Daedalus 109, 1: 121-136.

33. Steve Woolgar. 1990. Configuring the user: the case of usability trials. The Sociological Review 38, S1: 58-99. http://doi.org/10.1111/j.1467-954X.1990.tb03349.x
34. La Monte Young (ed.). 1963. An Anthology of Chance Operations. La Monte Young \& Jackson Mac Low.

35. Amit Zoran and Joseph A. Paradiso. 2013. FreeD: A Freehand Digital Sculpting Tool. Proceedings of the SIGCHI Conference on Human Factors in Computing Systems, ACM, 2613-2616.

http://doi.org/10.1145/2470654.2481361 